\title{
Androgen-receptor splice variant-7-positive prostate cancer: a novel molecular subtype with markedly worse androgen-deprivation therapy outcomes in newly diagnosed patients
}

\author{
Heng Li ${ }^{1,2,9}$, Zhize Wang ${ }^{1,2,9}$, Wei Xiao ${ }^{3}$, Libin Yan ${ }^{1,2}$, Wei Guan ${ }^{1,2}$, Zhiquan $\mathrm{Hu}^{1,2}$, \\ Lily $\mathrm{Wu}^{4,5}$, Qihong Huang ${ }^{6}$, Ji Wang ${ }^{7}$, Hua $\mathrm{Xu}^{1,2}, \mathrm{Xu}$ Zhang $^{8}$ and Zhangqun $\mathrm{Ye}^{1}$ \\ ${ }^{1}$ Department of Urology, Tongii Hospital, Tongji Medical College, Huazhong University of Science and \\ Technology, Wuhan, China; ${ }^{2}$ Hubei Institute of Urology, Tongji Hospital, Tongji Medical College, Huazhong \\ University of Science and Technology, Wuhan, China; ${ }^{3}$ Translational Medicine Center, Tongji Hospital, \\ Tongji Medical College, Huazhong University of Science and Technology, Wuhan, China; ${ }^{4}$ Department of \\ Molecular and Medical Pharmacology, David Geffen School of Medicine, University of California Los Angeles, \\ Los Angeles, CA, USA; ${ }^{5}$ Department of Urology, David Geffen School of Medicine at UCLA, Los Angeles, CA, \\ USA; ${ }^{6}$ The Wistar Institute, Philadelphia, PA, USA; ${ }^{7}$ Department of Cell Death and Cancer Genetics, the \\ Hormel Institute University of Minnesota, Austin, USA and ${ }^{8}$ Department of Urology/State Key Laboratory of \\ Kidney Diseases, Chinese PLA General Hospital/PLA Medical School, Beijing, China
}

\begin{abstract}
Androgen-deprivation therapy has been the standard treatment for metastatic and locally advanced prostate cancer, but the majority of patients will progress to castration-resistant prostate cancer within 2-3 years. Unlike the case in breast cancer, no clinically validated biomarker has been used to predict the outcomes of androgendeprivation therapy. To evaluate androgen-receptor splice variant-7 (AR-V7) detection in newly diagnosed advanced prostate cancer and describe the distinctive prognosis of this novel molecular subtype, this study retrospectively enrolled 168 newly diagnosed prostate cancer patients from 2003 to 2015 who received androgendeprivation therapy. AR-V7 immunohistochemical staining was performed with a monoclonal antibody, and ARV7 expression was determined using Immune-Reactive Score data. The association between nuclear AR-V7 expression and prognosis was determined. Multiple cause-specific Cox regression and stratified cumulative incidences were used to analyze the prognosis risk. Among the 168 patients, 32 (19\%) were AR-V7-positive. Compared with the AR-V7-negative patients, the AR-V7-positive patients had significantly lower prostate-specific antigen response rates $(P<0.001)$ to androgen-deprivation therapy and a much shorter time to castrationresistant prostate cancer $(P<0.0001)$. In Kaplan-Meier analysis, the AR-V7-positive group showed markedly lower castration-resistant prostate cancer progression-free survival $(P<0.0001)$ and much lower cancer-specific $(P<0.0001)$ and overall survival $(P<0.0001)$ both in all enrolled patients and in patients with metastases. AR-V7 positivity was a significant predictor of castration-resistant prostate cancer progression in multiple Cox regression (hazard ratio: 4.826; 95\% Cl: 2.960-7.869; $P<0.001$ ). AR-V7 immunohistochemical detection in newly diagnosed prostate cancer patients who are planning to receive androgen-deprivation therapy, especially those with metastases, is necessary and valuable for prognostic assessment. AR-V7-positive prostate cancer should be considered a novel prostate cancer subtype that should be distinguished upon initial biopsy. The main limitation of this study is its observational nature.
\end{abstract}

Modern Pathology (2018) 31, 198-208; doi:10.1038/modpathol.2017.74; published online 27 October 2017

Correspondence: Dr $\mathrm{H} \mathrm{Xu}$, Department of Urology, Tongji Hospital, Tongji Medical College, Huazhong University of Science and Technology, Wuhan 430030, China.

E-mail: xuhua@hust.edu.cn

${ }^{9}$ These authors contributed equally to this work.

Received 28 February 2017; accepted 15 May 2017; published online 27 October 2017
Since Huggins and Hodges ${ }^{1}$ first demonstrated the dependence of prostate cancer on androgen signaling in 1941, androgen-deprivation therapy through either medical or surgical castration has been the standard-of-care for metastatic and locally advanced disease. Androgen-deprivation therapy has been 
demonstrated to provide an initial benefit, but the majority of patients progress to castration-resistant prostate cancer within 2-3 years. ${ }^{2,3}$

Substantial improvements have been made in the treatment of prostate cancer, but unlike in breast cancer, ${ }^{4,5}$ clinically validated molecular subtypes of the disease are not yet used to guide patient management. More recently, the identification of androgen-receptor variants has been established as one of the mechanisms of castration-resistant prostate cancer progression. ${ }^{6-12}$ Among these variants, the androgen-receptor splice variant-7 (AR-V7) is the most abundant and best characterized..$^{6,8,13}$ Several pre-clinical and clinical trials have showed that the level of AR-V7 expression has a substantial relationship with resistance to enzalutamide, abiraterone, and other androgen-deprivation therapies, leading to poor prognosis and shorter survival times in castration-resistant prostate cancer, ${ }^{6,8-11,13-16}$ but there is no association with primary resistance to taxane or cabazitaxel. ${ }^{17,18}$

However, there are still no clear descriptions of the clinicopathological characteristics of newly diagnosed, AR-V7-positive prostate cancer. In this study, the clinical data of 168 advanced or metastatic prostate cancer patients who received androgendeprivation therapy at our hospital during the past 12 years were retrospectively collected. We determined the intensity of AR-V7 and androgen-receptor full-length (AR-FL)-positive staining in needle biopsies by immunohistochemistry and concluded that AR-V7-positive prostate cancer should be classified as a novel molecular subtype due to its worse response to androgen-deprivation therapy treatment, cancer-specific and overall survival compared with AR-V7-negative prostate cancer.

\section{Materials and methods}

\section{Patients and Tissue Samples}

The included patients had histologically confirmed prostate adenocarcinoma by needle biopsy, received standard androgen-deprivation therapy, including surgical (orchiectomy) or medical castration (luteinizing hormone releasing hormone analog), combined with or without medical anti-androgen (bicalutamide, etc), and had sufficient formalinfixed paraffin-embedded matched archival tissue for immunohistochemistry. Patients were excluded

Table 1 Clinicopathological characteristics of the patient cohort at diagnosis

\begin{tabular}{|c|c|c|c|c|}
\hline \multirow{2}{*}{ Characteristics } & \multicolumn{4}{|c|}{ Results } \\
\hline & Total & $A R$-V7 positive & $A R-V 7$ negative & $\mathrm{P}$-value $\mathrm{a}^{\mathrm{a}}$ \\
\hline No. of patients (\%) & 168 & 32 (19) & $136(81)$ & \\
\hline Age (median, range) & $71(53-96)$ & $71(58-79)$ & $72(53-96)$ & 0.09 \\
\hline Gleason score (n, \%) & & & & 0.026 \\
\hline$\leq 7$ & $60(35.7)$ & $6(18.8)$ & $54(39.7)$ & \\
\hline$\geq 8$ & $108(64.3)$ & $26(81.3)$ & $82(60.3)$ & \\
\hline T stage (n, \%) & & & & 0.398 \\
\hline$\leq \mathrm{T}_{2}$ & $16(9.5)$ & $2(6.3)$ & $14(10.3)$ & \\
\hline $\mathrm{T}_{3}$ & $49(29.2)$ & $8(25)$ & $41(30.1)$ & \\
\hline$\geq \mathrm{T}_{4}$ & $65(38.7)$ & $16(50)$ & $49(36)$ & \\
\hline $\mathrm{T}_{X}$ & $38(22.6)$ & $6(18.8)$ & $32(23.5)$ & \\
\hline$N$ stage (n, \%) & & & & 0.054 \\
\hline $\mathrm{N}_{0}{ }^{\circ}$ & $44(26.2)$ & $12(37.5)$ & $32(23.5)$ & \\
\hline$\geq \mathrm{N}_{1}$ & $76(45.2)$ & $10(31.3)$ & $66(48.5)$ & \\
\hline$\overline{\mathrm{N}}_{X}$ & $48(28.6)$ & $10(31.3)$ & $38(27.9)$ & \\
\hline$M$ stage (n, \%) & & & & 0.147 \\
\hline $\mathrm{M}_{0}$ & $36(21.4)$ & $4(12.5)$ & $32(23.5)$ & \\
\hline $\mathrm{M}_{1}$ & $118(70.2)$ & $26(81.3)$ & $92(67.6)$ & \\
\hline $\mathrm{M}_{X}$ & $14(8.3)$ & $2(6.3)$ & $12(8.8)$ & \\
\hline Total prostate-specific antigen (ng/ml) & $118.78(2.61-4003.40)$ & $221.04(34.09-2507.10)$ & $113.40(2.61-4003.40)$ & 0.405 \\
\hline Free prostate-specific antigen $(\mathrm{ng} / \mathrm{ml})$ & $19.18(0.12-640.19)$ & $44.68(3.58-640.19)$ & $15.17(0.12-280.51)$ & 0.093 \\
\hline $\begin{array}{l}\text { Free prostate-specific antigen/total } \\
\text { prostate-specific antigen }(\%)\end{array}$ & $13.93(1.89-64.44)$ & $17.70(4.85-44.66)$ & $13.25(1.89-66.44)$ & 0.190 \\
\hline Prostate volume $\left(\mathrm{cm}^{3}\right)$ & $106.49(31.45-719.71)$ & $100.42(40.26-172.80)$ & $108.51(31.45-719.71)$ & 0.007 \\
\hline Prostate-specific antigen density $\left(\mathrm{ng} / \mathrm{ml} / \mathrm{cm}^{3}\right)$ & $1.18(0.02-62.63)$ & $3.36(0.38-23.90)$ & $0.97(0.02-62.63)$ & 0.698 \\
\hline
\end{tabular}

${ }^{a} P$-values are based on Fisher's exact test and Student's $t$-test for categorical and continuous variables, respectively.

Bold values signify the $P$-value $\leq 0.05$. 
if they received additional concurrent anticancer therapies (radical prostatectomy, chemotherapy or radiotherapy), did not complete the androgendeprivation therapy or had other severe diseases. Demographic and clinical data for each patient were retrospectively collected from the hospital electronic patient record system.

We retrospectively collected 209 prostate cancer patients who were diagnosed with metastatic or locally advanced prostate cancer and began first line androgen-deprivation therapy at the Department of Urology, Tongji Hospital of Huazhong University of Science and Technology between the years 2003 and 2015. Among these patients, 41 were excluded because of additional concurrent anticancer therapies or nonstandard androgen-deprivation therapy (Supplementary Figure 1; Table 1). In total, 168 patients were enrolled in our study. The median time from diagnosis to data analysis was 36 months (IQR: 22-48 months).

\section{Study Design and Assessments}

This was a retrospectively study evaluating the ability of baseline (pretreatment) AR-V7 status (positive vs negative) by immunohistochemistry to predict prostate-specific antigen response and risk of castration-resistant prostate cancer progression following androgen-deprivation therapy in primary prostate cancer. This study was registered in the Chinese Clinical Trial Registry (NO ChiCTR-POC-16008239, http://www.chictr.org.cn/), and carried out in accordance with the ethical standards of the Helsinki Declaration and approved by the Tongji Hospital of Huazhong University of Science and Technology (Wuhan, China) ethics review committee (reference TJ-IRB20160308). Written informed consent was obtained from each patient prior to any study-specific investigations.

Follow-up assessments were retrospectively collected and included prostate-specific antigen (PSA) measurements, prostate ultrasound scans, computed tomography (CT) of the chest, abdomen and pelvis, and technetium-99 $\mathrm{m}$ bone scanning. Clinical investigators were unaware of the AR-V7 status of the participants. All immunohistochemical slides were examined and scored by two experienced pathologists, who were blinded to all clinical data. If the Immune-Reactive Score differed between the investigators, a third investigator evaluated the tissue sections, and the average score was recorded.

\section{Clinical Outcomes}

The primary endpoints were freedom from PSA and clinical or radiographic progression. PSA progression was defined as an increase in the PSA level by $25 \%$ or more above the nadir (and by $\geq 2 \mathrm{ng} / \mathrm{ml}$ ), with confirmation four or more weeks later (PCWG3 criteria). ${ }^{19,20}$ Clinical or radiographic progression was defined as symptomatic progression (worsening disease-related symptoms or new cancer-related complications), or $\geq 20 \%$ increase in the sum of the diameters of the soft-tissue target lesions on CT scanning (according to the Response Evaluation Criteria in Solid Tumors ${ }^{21}$ ), or $\geq 2$ new bone lesions on bone scans, or death, whichever occurred first. ${ }^{19}$ Time to castration-resistant prostate cancer was defined as the time from diagnosis to biochemical and/or radiological progression with castrate serum level of testosterone. We also recorded the proportion of patients with a PSA response (PSA level $\leq 4 \mathrm{ng} / \mathrm{ml}$, maintained for $\geq 4$ weeks) at 6 and 7 months of treatment and the best PSA response (maximal percentage decrease in PSA level from baseline) for each patient. ${ }^{15,22}$

Secondary endpoints were cancer-specific and overall survival, which were defined as the time to death due to prostate cancer and any cause, respectively.

\section{Histopathological Assessments and Immunohistochemistry}

Immunohistochemistry was uniformly performed to assess protein expression of AR-V7 (rabbit monoclonal, ab198394, Abcam, Cambridge, UK, 1:100 dilution ), AR-FL (rabbit polyclonal, ab74272, Abcam, 1:200 dilution), PSA (mouse monoclonal, ZM-0218, ZSGB-bio, Beijing, PRC, 1:150 dilution), p504s/AMACR (rabbit monoclonal, ZA-0227, ZSGBbio, 1:200 dilution), p63 (mouse monoclonal, MAB-0365, Maixin-Bio, Fuzhou, PRC, 1:200 dilution), HMW (mouse monoclonal, ZM-0308, ZSGB-bio, 1:200 dilution) by Bond Polymer Refine Detection System (Leica Biosystems Newcastle, Newcastle upon Tyne, UK). Omission of the primary antibody with phosphate-buffered saline served as a negative control for this detection system. We also performed immunohistochemical staining of liver carcinoma/ para-carcinoma tissues and benign prostate hypertrophy tissues as negative controls for the antibody (Supplementary Figures 2, 3, 4 and 5).

Considering the heterogeneity of prostate cancer, we selected tumor regions where AR-V7 expression was strongest to evaluate each tissue sample. To evaluate prostate nuclear AR-V7 expression, we implemented an Immune-Reactive Score that included the intensity and quantity of cells stained as previously reported. ${ }^{14,23}$ Immune-reactive scores of 0 or 1 were considered negative and scores $\geq 2$ were considered positive. ${ }^{14} \mathrm{~A}$ more detailed immunohistochemical protocol and the raw data of Immune-Reactive Score were given in the Supplement.

\section{Antibody Specificity}

The specificity of the anti-AR (ab74272, Abcam) and monoclonal anti-AR-V7 antibodies was determined 
$\mathrm{H} \& \mathrm{E}$
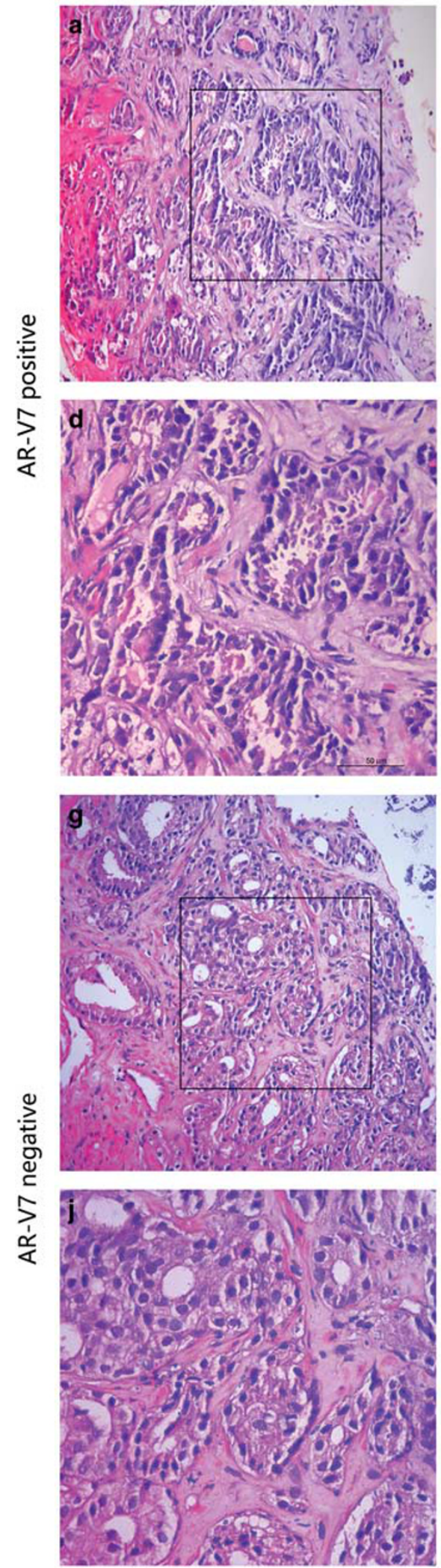

AR-FL
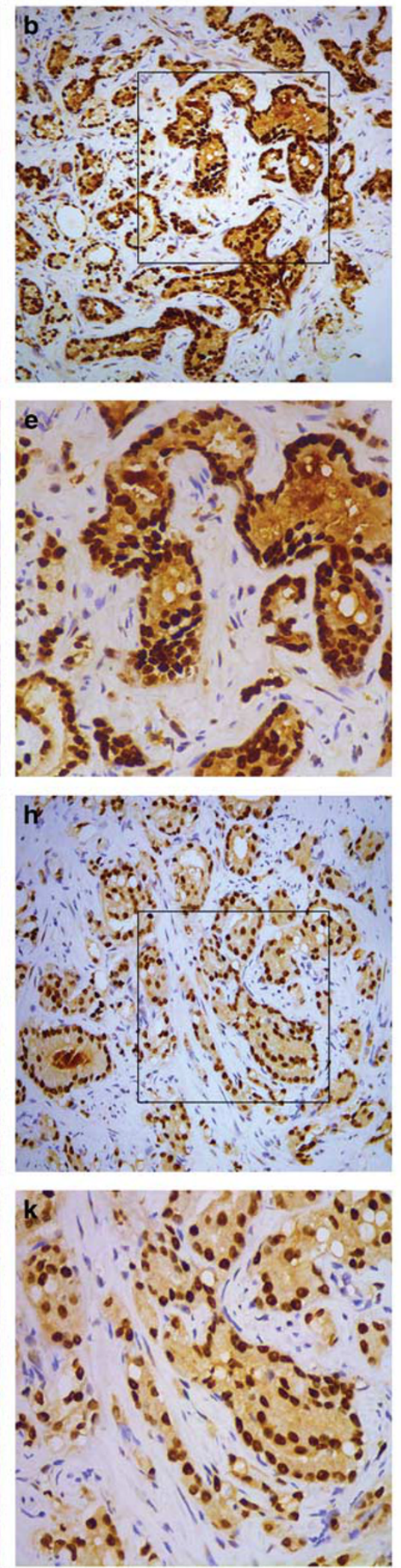

AR-V7

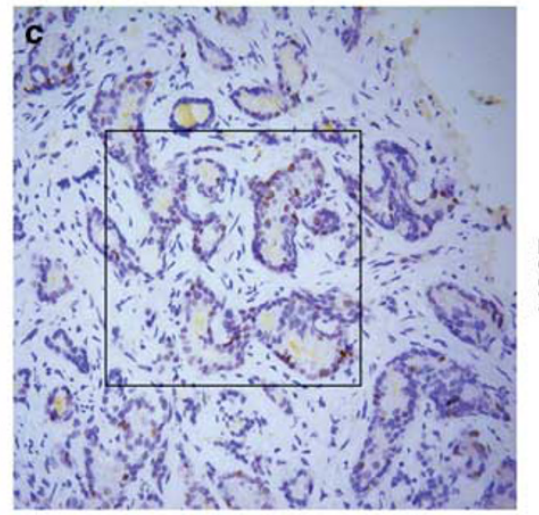

๙ัญ

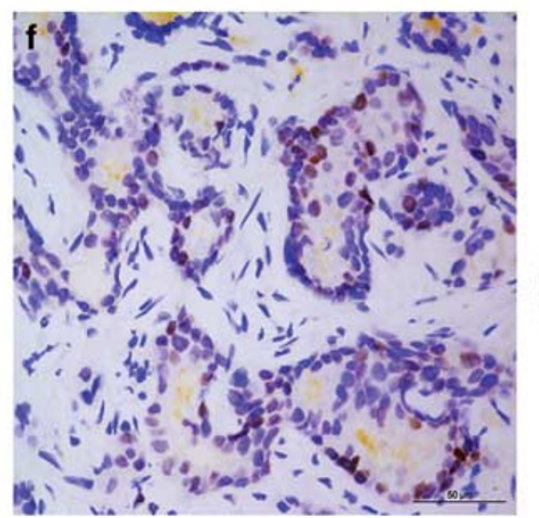

을

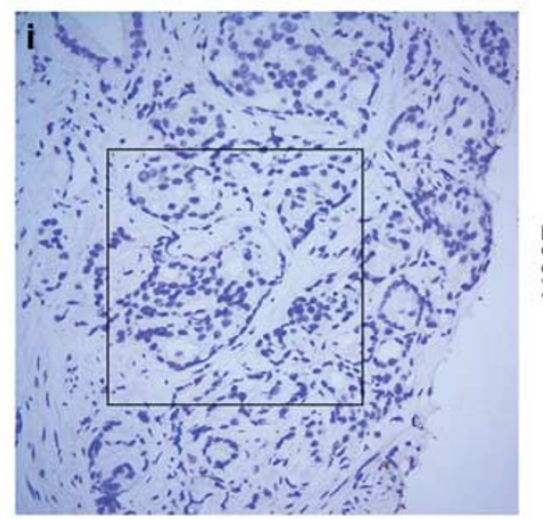

กับ

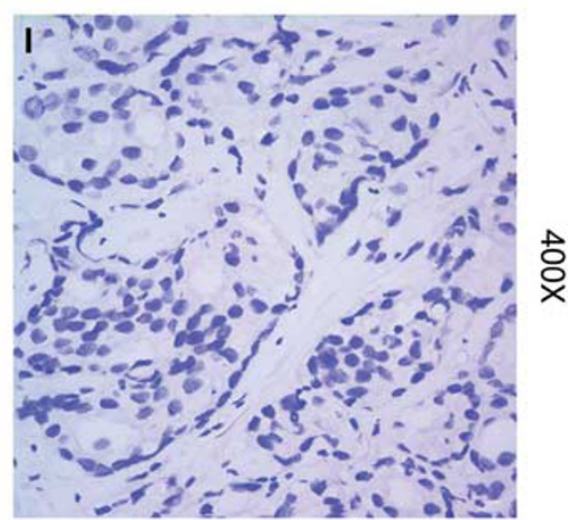

Figure 1 Representative immunohistochemical (IHC) and hematoxylin-eosin (H\&E) staining. (a, d, g, j) H\&E staining; (b, e, h, k) immunohistochemical staining for N-terminal androgen-receptor full-length (AR-FL) and (c, f, i, l) immunohistochemical staining for ARV7 in two representative biopsy cores. The first and second rows are AR-V7-positive from consecutive tissue sections, and the third and fourth rows are AR-V7-negative. Original magnification: $\times 200(\mathbf{a}, \mathbf{b}, \mathbf{c}, \mathbf{g}, \mathbf{h}, \mathbf{i})$ and $\times 400(\mathbf{d}, \mathbf{e}, \mathbf{f}, \mathbf{j}, \mathbf{k}, \mathbf{l})$. 
by western blotting using the prostate cancer cell lines PC3, LNCaP (Stem Cell Bank, Chinese Academy of Sciences, Shanghai, China) and 22Rv1 (Boster Biological Engineering, Wuhan, China). Of note, the anti-AR antibody showed a number of nonspecific bands, but the AR-V7 antibody showed a relatively specific band around $76 \mathrm{kDa}$ in 22Rv1 cells, which are known to be AR-V7 positive (Supplementary Figure 12). To further demonstrate the ability of the antibody to detect AR-V7, LNCaP and 22Rv1 cells were transfected with siRNAs targeting AR-exon 1, AR-exon 7, AR-V7 CE3b, or siRNA-negative control. ${ }^{16}$ Suppression of AR-V7 protein was observed with siRNAs to exon 1 and CE3, but not the negative control siRNA or exon 7, an exon absent from the AR-V7 mRNA (Supplementary Figure 13; Supplementary Table 7). Overall, these data indicate that the monoclonal antibody binds specifically to AR-V7.

\section{Statistical Analyses}

Continuous data are presented as median (range) and categorical data are presented as number (proportion). Patient clinicopathological characteristics according to AR-V7 expression were compared using the Student's $t$-test for continuous variables and the $\chi^{2}$ test for categorical variables. Univariate and multivariate Cox regression models were used to evaluate the predictive role of all covariates for the development of castration-resistant prostate cancer. PSA-free progression, time to castration-resistant prostate cancer progression, cancer-specific, and overall survival were estimated using the KaplanMeier method and compared by the log-rank test, patients with metastases $\left(\mathrm{N}_{1}\right.$ or $\mathrm{M}_{1}$ stage) were stratified for further analysis. Statistical analyses were performed using SPSS v.22 (IBM, Armonk, NY, USA) and Prism v.6 (GraphPad, La Jolla, CA, USA).

\section{Results}

\section{Patient Characteristics}

Table 1 lists patient baseline characteristics; 32 patients (19\%) were AR-V7-positive and 136 (81\%) were AR-V7-negative. The AR-V7-positive patients had a higher Gleason score $(P=0.026)$ and smaller prostate volume $(P=0.007)$ compared with AR-V7negative patients.

\section{Immunohistochemical Pathological Characteristics of AR-V7-Positive Prostate Cancer}

The proportion of AR-V7-positive prostate cancer was $19 \%$ in our study. Of the AR-V7-positive patients, 10 were strongly positive (Immune-Reactive Score $\geq 9$ ), whereas 22 were moderately positive $(2 \leq$ Immune-Reactive Score $<9)$ (Supplementary Figures 6, 7, 8, 9 and 10).

Representative immunohistochemical staining was shown in Figure 1. The AR-V7 monoclonal antibody exhibited high specificity with no crossreaction to full-length androgen receptors. From the AR-V7-positive sections, we observed the following characteristics: (1) AR-V7 was primarily expressed in the nuclei of luminal epithelial cells in malignant prostate glands; (2) AR-V7 expression was dispersed in some malignant glands but not all, and the proportion of AR-V7-expressing cells in positive glands ranged from 15 to $80 \%$ (Supplementary Figure 6); and (3) blood vessel and stroma cells were AR-V7-negative, which could be used as an internal control for the immunohistochemistry.

\section{The PSA Response Rate is Much Lower in AR-V7- Positive Patients After Androgen-Deprivation Therapy}

The overall proportion of patients who had PSA responses during androgen-deprivation therapy was

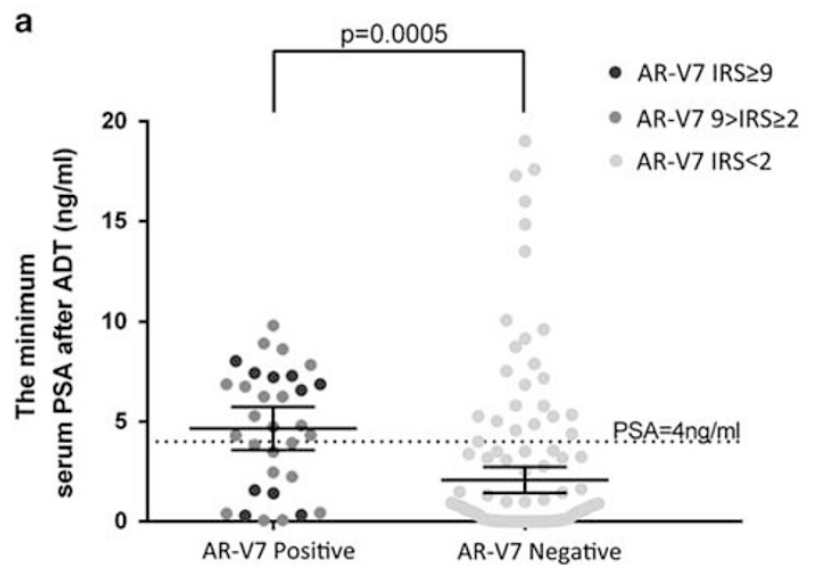

b

b

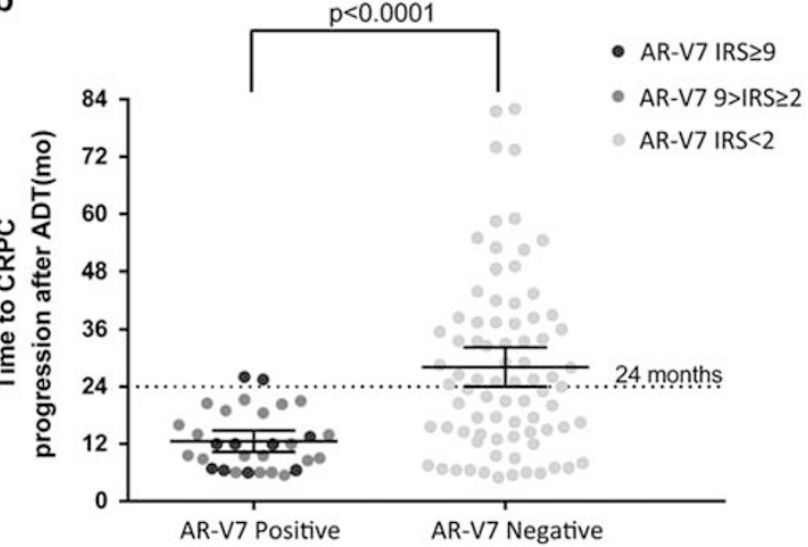

Figure 2 (a) Minimum serum prostate-specific antigen (PSA) level after androgen-deprivation therapy (ADT) for strongly positive AR-V7 (Immune-Reactive Score $\geq$ 9, black plot), moderately positive AR-V7 (9>Immune-Reactive Score $\geq 2$, gray plot) and AR-V7-negative (Immune-Reactive Score <2, light gray plot). Black crosshairs denote the median PSA with 95\% CI. (b) The time to castration-resistant prostate cancer (CRPC) progression after androgen-deprivation therapy in the AR-V7-positive and AR-V7-negative groups. Black crosshairs denote the median time to CPRC progression with 95\% CI. P-values are for Student's $t$-tests. 
$74 \%$ (125 of 168). The PSA response rate among ARV7-positive patients was $41 \%$ (13 of 32 ), whereas the rate among AR-V7-negative patients was $82 \%$ (112 of 136) $(P<0.001)$. The median minimum PSA of ARV7-positive patients after androgen-deprivation therapy was $4.65 \mu \mathrm{g} / \mathrm{ml}$, whereas in AR-V7-negative patients it was $2.08 \mu \mathrm{g} / \mathrm{ml}(P=0.0005)$ (Figure 2a). The median best PSA response rate of AR-V7positive and -negative patients after androgendeprivation therapy was 99 and $100 \%$, respectively $(P=0.3156)$ (Supplementary Figure 11).

\section{Primary Endpoints}

During the surveillance, 109 patients progressed to castration-resistant prostate cancer with a median time of 19 month (range: 5-82 months). The median time to castration-resistant prostate cancer in AR-V7positive patients was 12 months (range: 626 months), whereas 25 months (range: 5-82, $P<0.0001$ ) in AR-V7-negative patients (Figure 2b). The 2-year cumulative incidence of castrationresistant prostate cancer progression was $88 \%$ (95\% CI: $75-100 \%$ ) in the AR-V7-positive group compared with $28 \%$ (95\% CI: $20-36 \%$ ) in the ARV7-negative group (Figure 2b). Kaplan-Meier analyses indicated a significant reduction in PSA and castration-resistant prostate cancer progression-free survival in AR-V7-positive patients, as in the stratified patients with metastases (both $P<0.0001$ ) (Figures 3a, b, e and f).

\section{Secondary Endpoints}

In total, 44 of 168 patients died of prostate cancer $(26 \%)$. The proportion of prostate cancer-related deaths among AR-V7-negative patients was $22 \%$ (30 of 136), whereas 44\% (14 of 32) AR-V7-positive patients died of cancer-related causes, which was nearly double the proportion in the AR-V7-negative group $(P=0.012)$. Kaplan-Meier analyses indicated that cancer-specific and overall survival were dramatically lower in AR-V7-positive patients, as in the stratified patients with metastases (both $P<0.0001$ ) (Figures 3c, d, g and h).

\section{AR-V7 is a Risk Factor for Castration-Resistant Prostate Cancer Progression and Cancer-Specific and Overall Survival}

Univariate cause-specific Cox proportional hazard regression models indicated that AR-V7 positivity $(P<0.001), \quad \mathrm{T}$ stage $(P=0.001), \quad$ Gleason score $(P=0.005)$, M stage $(P=0.024)$, free prostate-specific antigen level $(P=0.02)$, and age at diagnosis $(P=0.038)$ were significant risk factors of shorter progression time to castration-resistant prostate cancer. Multivariate cause-specific Cox proportional hazard regression models indicated that AR-V7 positivity (ref: negative, HR: 4.826; $P<0.001$ ),
Gleason score (ref: $\leq 7$ HR: 1.616, $P=0.024$ ), and T stage (ref: $\leq 2$ HR: 2.999, $P=0.004$ ) were independent predictors of time to castration-resistant prostate cancer progression after adjusting for age, M stage, and free prostate-specific antigen at diagnosis (Table 2). Multivariate cause-specific Cox proportional hazard regression model also indicated that AR-V7 positivity (ref: negative, HR: $4.339 ; P<0.001$ ), Gleason score (ref: $\leq 7$ HR: 2.068, $P=0.038$ ) and PSA density (ref: $\leq 1.18 \mathrm{ng} / \mathrm{ml} / \mathrm{cm}^{3}$, HR: $2.134, P=0.025$ ) were independent predictors of cancer-specific survival (Supplementary Table 2). More interestingly, AR-V7 positivity (ref: negative, HR: 3.976; $P<0.001$ ) was the only independent predictor of overall survival (Supplementary Table 3).

The association of results from the model that omitted information regarding AR-V7 status were similar to those from the stratified model (Supplementary Table 4). Risk reclassification plots showed AR-V7 status at diagnosis significantly increased the predicted risk of castration-resistant prostate cancer progression in the 1st, 2nd, 3rd and 7th year of surveillance beyond age, free prostatespecific antigen, $\mathrm{T}$ stage, $\mathrm{M}$ stage, and Gleason score (Figure 4).

\section{Routine Immunohistochemical Indicators Could not Predict the Risk of Castration-Resistant Prostate Cancer Progression}

There was no statistical difference between routine immunohistochemical indicators including PSA, P504s, P63, and HMW in the AR-V7-positive and -negative subgroups. Compared with AR-V7, routine immunohistochemical indicators could not predict castration-resistant prostate cancer progression (Supplementary Table 5).

\section{Discussion}

Androgen-deprivation therapy has been the standard-of-care for metastatic and locally advanced prostate cancer, but the majority of patients progress to castration-resistant prostate cancer within 2-3 years. $^{2,3}$ Therefore, it is clinically necessary to identify patients who will have a poor reaction to androgen-deprivation therapy and rapidly progress to castration-resistant prostate cancer. Although patients were retrospectively enrolled, this study demonstrated that $<50 \%$ of AR-V7-positive prostate cancer patients have PSA responses to androgendeprivation therapy and almost $90 \%$ will progress to castration-resistant prostate cancer within 2 years, which was statistically different from AR-V7negative patients. Furthermore, despite having only 186 enrolled patients, Kaplan-Meier analyses indicated significant differences in cancer-specific $(P<0.0001)$ and overall survival $(P<0.0001)$ according to AR-V7 status, and similar results were concluded in the stratified patients with metastases 
a

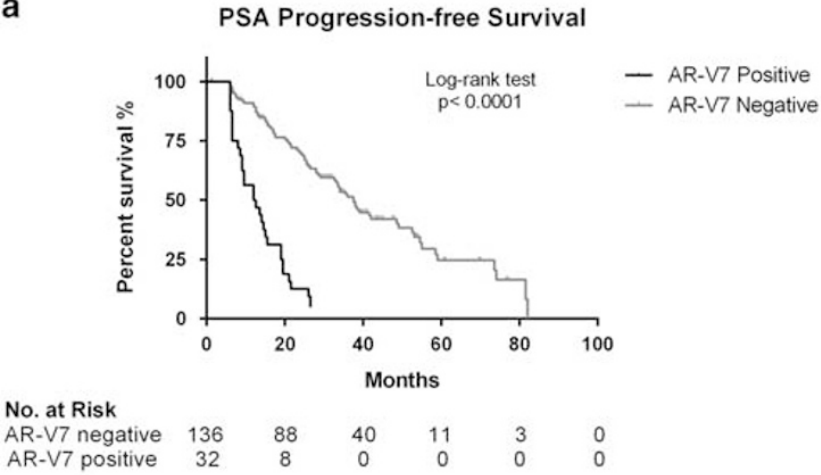

C

Cancer Specific Survival

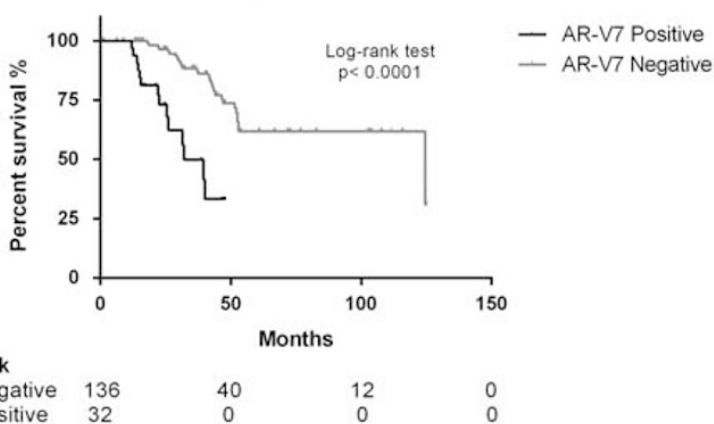

No. at Risk

$\begin{array}{lcccc}\text { AR-V7 negative } & 136 & 40 & 12 & 0 \\ \text { AR-V7 positive } & 32 & 0 & 0 & 0\end{array}$

e PSA Progression-free Survival of patients with metastases

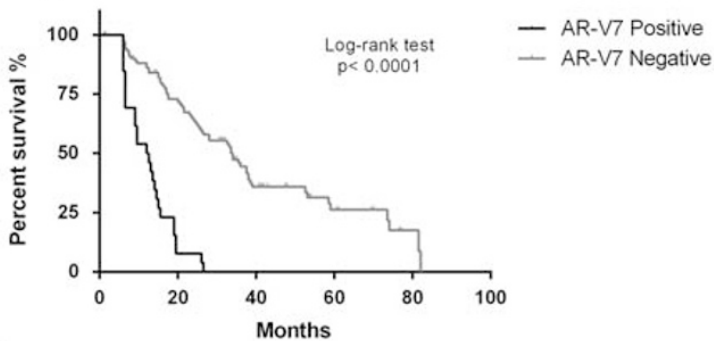

No. at Risk

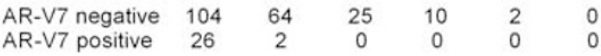

g

Cancer Specific Survival of patient with metastases

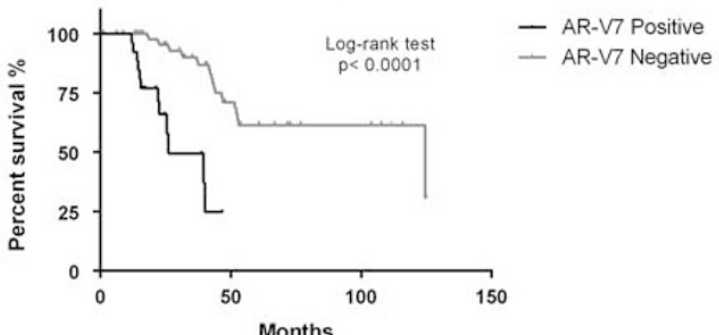

No. at Risk

$\begin{array}{lccll}\text { AR-V7 negative } & 104 & 31 & 9 & 0 \\ \text { AR-V7 positive } & 26 & 0 & 0 & 0\end{array}$

(both $P<0.0001)$. These results show that AR-V7 detection in newly diagnosed prostate cancer patients, especially those with metastases, who will receive androgen-deprivation therapy, is valuable and necessary for prognostic assessment. b

CRPC Progression-free Survival

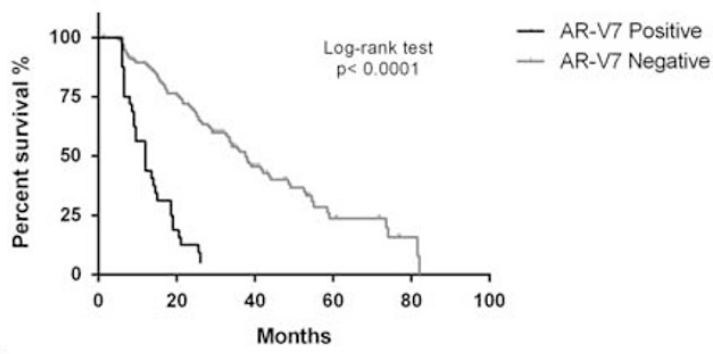

No. at Risk

$\begin{array}{lcccccc}\text { AR-V7 negative } & 136 & 88 & 42 & 10 & 3 & 0\end{array}$

d

Overall Survival

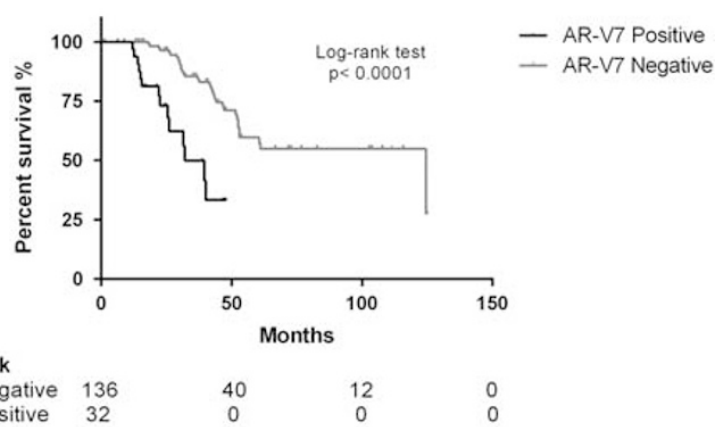

No. at Risk

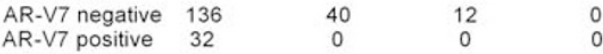

f CRPC Progression-free Survival of patient with metastases

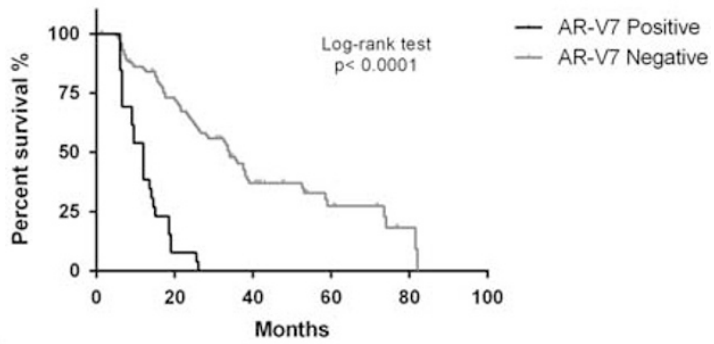

No. at Risk

$\begin{array}{lcccccc}\text { AR-V7 negative } & 104 & 63 & 27 & 10 & 2 & 0 \\ \text { AR-V7 positive } & 26 & 2 & 0 & 0 & 0 & 0\end{array}$

h

Overall Survival of patient with metastases

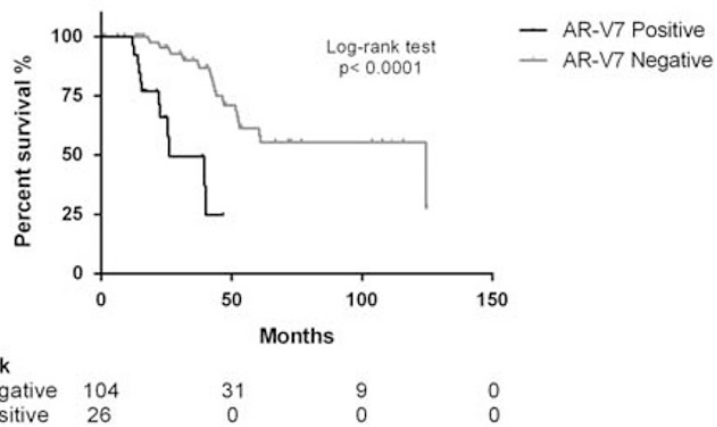

$\mathrm{AR}-\mathrm{V} 7$ is a truncated androgen-receptor protein that lacks the C-terminal ligand-binding domain but retains the trans-activating $\mathrm{N}$-terminal domain. , $^{6,7}$ Although the resultant truncated protein is unable to bind ligand, it is constitutively active as a 
Figure 3 Kaplan-Meier analysis of prostate-specific antigen (PSA) progression-free, castration-resistant prostate cancer (CRPC) progression- free, cancer-specific, and overall survival according to AR-V7 status in all enrolled patients (a-d) and in the patients with metastases (e-h). (a) The median PSA progression-free survival in AR-V7-positive patients was 13 months (95\% CI: 6.956-18.044), whereas in AR-V7-negative patients it was 38 months (95\% CI: 32.685-42.315), the hazard ratio for PSA progression with AR-V7 positivity was 5.435; 95\% CI (3.374-8.754; $P<0.0001$ by the log-rank test). (b) The median time to castration-resistant prostate cancer progression in AR-V7-positive patients was 12 months (95\% CI: 8.562-15.438), whereas in AR-V7-negative patients it was 38 months (95\% CI: 31.14142.859), the hazard ratio for castration-resistant prostate cancer progression with AR-V7 positivity was 5.571 (95\% CI: 3.445-9.007; $P<0.0001$ by the log-rank test). (c) The median cancer-specific survival time in AR-V7-positive patients was 32 months (95\% CI: $19.879-$ 44.121), whereas in AR-V7-negative patients it was 125 months (95\% CI: 24.426-224.574), the hazard ratio for cancer-specific survival with AR-V7 positivity was 5.068 (95\% CI: 2.556-10.049; $P<0.0001$ by the log-rank test). (d) The median overall survival time in AR-V7positive patients was 32 months (95\% CI: 19.879-44.121), whereas in AR-V7-negative patients it was 125 months (95\% CI: 35.300213.700), the hazard ratio for overall survival with AR-V7 positivity was 4.667 (95\% CI: $2.382-9.142 ; P<0.0001$ by the log-rank test). (e) The median PSA progression-free survival in AR-V7-positive patients with metastases was 12 months (95\% CI: 8.002-15.998), whereas in AR-V7-negative patients it was 34 months (95\% CI: 26.244-41.756), the hazard ratio for PSA progression with AR-V7 positivity in patients with metastases was 5.879 (95\% CI: 3.480-9.931; $P<0.0001$ by the log-rank test). (f) The median time to castration-resistant prostate cancer progression in AR-V7-positive patients with metastases was 12 months (95\% CI: 9.569-14.431), whereas in AR-V7-negative patients it was 34 months (95\% CI: 26.855-41.145), the hazard ratio for castration-resistant prostate cancer progression with AR-V7 positivity in patients with metastases was 5.899 (95\% CI: 3.476-10.012; $P<0.0001$ by the log-rank test). (g) The median cancer-specific survival time in AR-V7-positive patients with metastases was 26 months (95\% CI: 9.196-42.804) whereas in AR-V7-negative patients it was 125 months (95\% CI: 24.166-224.834), the hazard ratio for cancer-specific survival with AR-V7 positivity patients with metastases was 6.122 (95\% CI: $2.847-13.164 ; P<0.0001$ by the log-rank test). (h) The median overall survival time in AR-V7-positive patients with metastases was 26 months (95\% CI: 9.196-42.804), whereas in AR-V7-negative patients it was 125 months (95\% CI: 34.957-214.043), the hazard ratio for overall survival with AR-V7 positivity patients with metastases was 6.122 (95\% CI: $2.847-13.164 ; P<0.0001$ by the logrank test).

Table 2 Adjusted cause-specific Cox proportional hazard regression analyses for time to castration-resistant prostate cancer

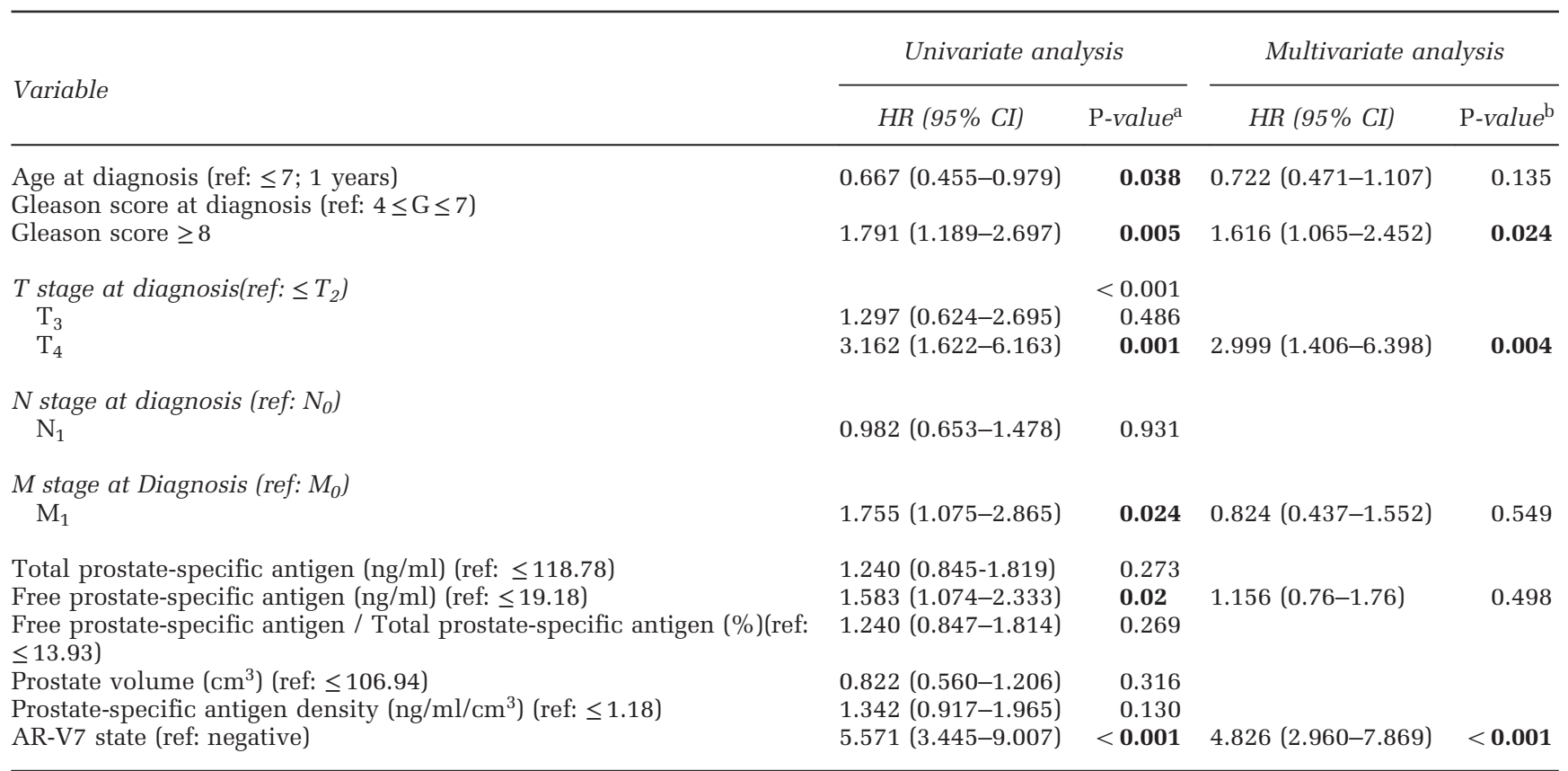

andividual covariates were tested to predict outcome using a univariate Cox proportional hazards model. ${ }^{\mathrm{b}} P$-values are the result of a adjusted multivariable Cox proportional hazards model.

Bold values signify the $P$-value $\leq 0.05$.

transcription factor and capable of promoting activation of target genes irrespective of androgen level, which promotes the development and growth of prostate cancer. ${ }^{6-12}$ This mechanism is consistent with the lower PSA response to androgendeprivation therapy in AR-V7-positive patients. ARV7-positive patients distinguished by immunohistochemistry rarely show a PSA response after androgen-deprivation therapy and quickly progress to castration-resistant prostate cancer within two years; however, AR-V7-negative patients have better long-term responses and benefits from androgendeprivation therapy. As far as we know, the biomarkers that are currently detected in the pathological diagnosis of prostate cancer (PSA, P504S, P63, and HMW) do not predict androgen-deprivation therapy responses or the risk of castration-resistant prostate cancer progression.

Prostate cancer is a biologically heterogeneous disease with variable molecular alterations underlying its initiation and progression. $^{24}$ Despite recent advances in our understanding of prostate cancer 
a

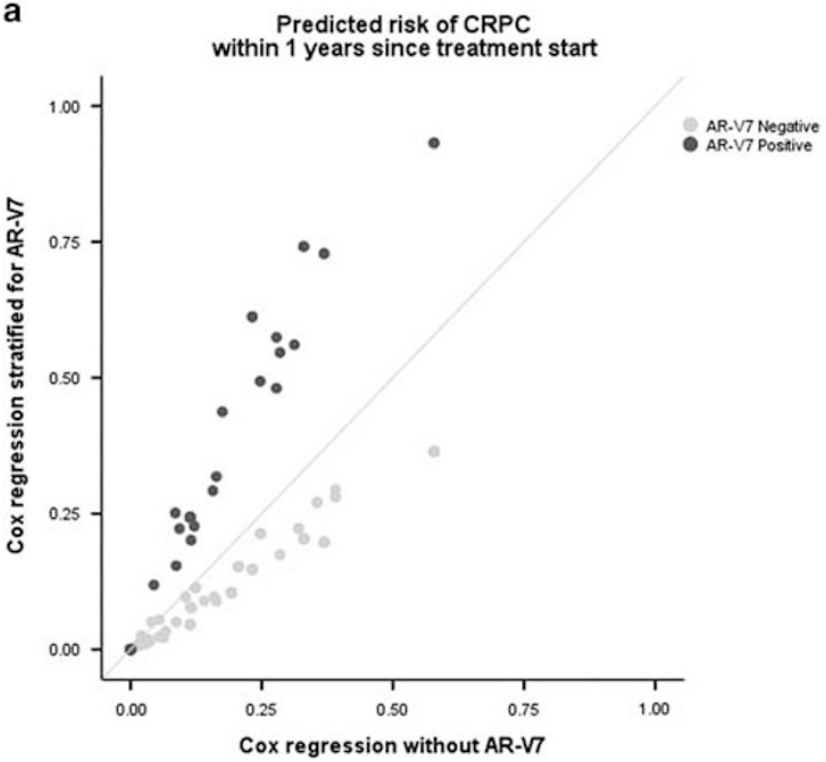

C

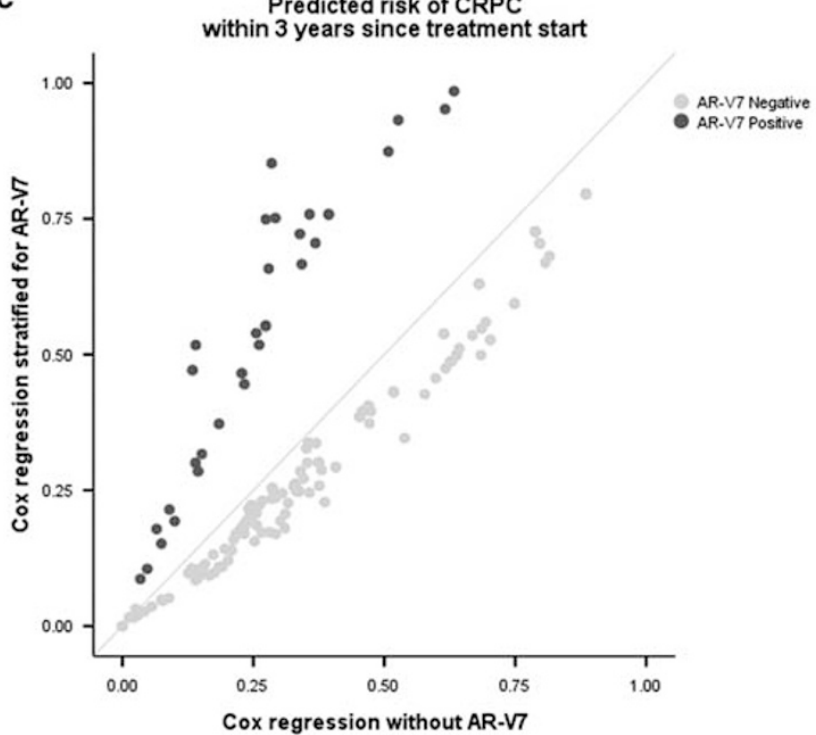

b

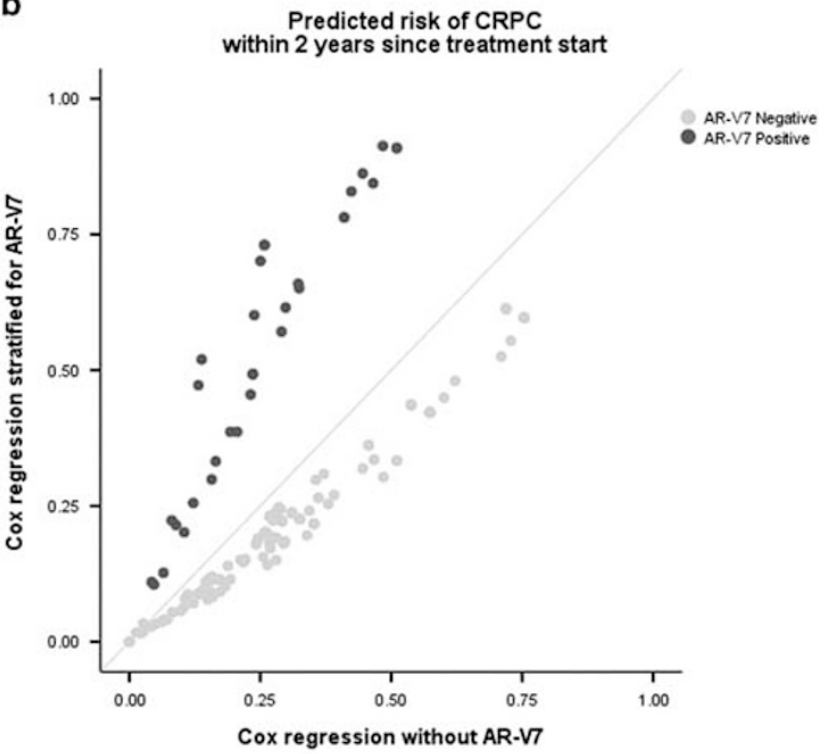

d

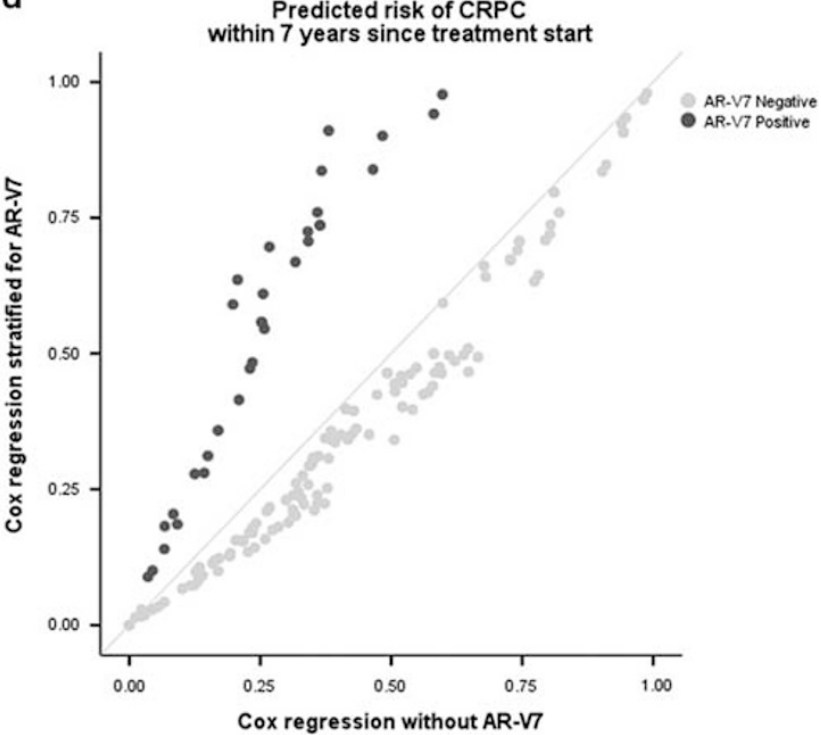

Figure 4 The effect of AR-V7 status on castration-resistant prostate cancer (CRPC) prognostication was compared for predictions obtained with a stratified multiple cause-specific Cox regression model to predictions obtained with a second multiple Cox regression model omitting AR-V7 status. The scatter plots show the predicted risk of castration-resistant prostate cancer within 1, 2, 3, and 7 years after beginning androgen-deprivation therapy for AR-V7-positive (black) and AR-V7-negative patients (gray).

heterogeneity, better methods for classifying prostate cancer are still needed to improve prognostic accuracy and therapeutic outcomes. The detection of AR-V7 in circulating tumor cells from patients with castrationresistant prostate cancer may be associated with resistance to enzalutamide and abiraterone, ${ }^{15}$ but is not associated with primary resistance to taxane or cabazitaxel chemotherapy. ${ }^{17,18}$ In this study, compared to circulating tumor cells detection, we detected ARV7 in biopsy samples by immunohistochemistry, which is significantly more economically and technically feasible in routine clinical practice. ${ }^{14,16}$ Different from the previously reported AR-V7 antibody, ${ }^{6,13}$ the AR-V7 monoclonal antibody used in this study showed high specificity with no cross-reactivity to full-length AR, which means this AR-V7 antibody is reliable and could be widely used in clinical settings. A systematic review of the existing clinical data (Supplementary Table 6) showed that the proportion of AR-V7 positivity in newly diagnosed patients detected by immunohistochemistry ranged from 17 to $40 \%,{ }^{6,14,16}$ which was consistent with our study $(19 \%)$, whereas the range was from 41 to $61 \%^{7,9}$ by RT-PCR. AR-V7 positive prostate cancer is a novel molecular subtype with distinctive clinical and pathological characteristics.

The main limitation of our study was its retrospective and single-institution, including those 
inherent to its observational nature. The median follow-up time from diagnosis to data analysis was 36 months (IQR: $22-48$ months), which is limited to observe the primary endpoint (109 of 168 patients progressed to castration-resistant prostate cancer) and the secondary endpoint (44 patients died of prostate cancer). The restrictive amount of endpoint events could raise serious concerns about the statistical power and utility of the multivariate analysis. More prospective, multicenter, large-scale trials are warranted to verify these results. In addition, our patients cohort were recruited in a long period, during which the androgen-deprivation therapy for prostate cancer were various (138 received maximal androgen blockade, 30 received castration). We stratified the patients into different androgen-deprivation therapy groups, and calculate the log-rank $P$-value by Kaplan-Meier method to analysis the AR-V7 predictive value for time to castration-resistant prostate cancer progression in similar primary androgen-deprivation therapy treatment patients (Supplementary Table 1).

In conclusion, our data indicate that AR-V7 expression in newly diagnosed prostate cancer is intimately correlated with prognosis and effectiveness of androgen-deprivation therapy. It is valuable and necessary to routinely detect AR-V7 by immunohistochemistry in newly diagnosed prostate cancer patients, especially those with metastases, before androgen-deprivation therapy administration.

\section{Acknowledgments}

This work was supported by the National Natural Science Foundation of China (Grant Numbers 31372562, 81470935, 81402098, 81402105, and 81402087), the Chenguang Program of Wuhan Science and Technology Bureau (Grant Number 2015070404010199), and The National High Technology Research and Development Program 863 (Grant Number 2014AA020607). We thank all investigators and staff at the participating institutions, team members of the pathology department of Tongji Hospital and, above all, the patients, without whom this work would not have been possible.

\section{Disclosure/conflict of interest}

The authors declare no conflict of interest.

\section{References}

1 Huggins C, Hodges CV. Studies on prostatic cancer. I. The effect of castration, of estrogen and androgen injection on serum phosphatases in metastatic carcinoma of the prostate. CA Cancer J Clin 1972;22: 232-240.

2 Harris WP, Mostaghel EA, Nelson PS, et al. Androgen deprivation therapy: progress in understanding mechanisms of resistance and optimizing androgen depletion. Nat Clin Pract Urol 2009;6:76-85.

3 Chandrasekar T, Yang JC, Gao AC, et al. Mechanisms of resistance in castration-resistant prostate cancer (CRPC). Transl Androl Urol 2015;4:365-380.

4 DeSantis C, Ma J, Bryan L, et al. Breast cancer statistics, 2013. CA Cancer J Clin 2014;64:52-62.

5 McLendon R, Friedman A, Bigner D, et al. Comprehensive genomic characterization defines human glioblastoma genes and core pathways. Nature 2008;455: 1061-1068.

6 Guo Z, Yang X, Sun F, et al. A novel androgen receptor splice variant is up-regulated during prostate cancer progression and promotes androgen depletionresistant growth. Cancer Res 2009;69:2305-2313.

$7 \mathrm{Hu}$ R, Dunn TA, Wei S, et al. Ligand-independent androgen receptor variants derived from splicing of cryptic exons signify hormone-refractory prostate cancer. Cancer Res 2009;69:16-22.

8 Sun S, Sprenger CC, Vessella RL, et al. Castration resistance in human prostate cancer is conferred by a frequently occurring androgen receptor splice variant. J Clin Invest 2010;120:2715-2730.

9 Hornberg E, Ylitalo EB, Crnalic S, et al. Expression of androgen receptor splice variants in prostate cancer bone metastases is associated with castration-resistance and short survival. PLoS ONE 2011;6:e19059.

10 Dehm SM, Schmidt LJ, Heemers HV, et al. Splicing of a novel androgen receptor exon generates a constitutively active androgen receptor that mediates prostate cancer therapy resistance. Cancer Res 2008;68: 5469-5477.

11 Watson PA, Chen YF, Balbas MD, et al. Constitutively active androgen receptor splice variants expressed in castration-resistant prostate cancer require full-length androgen receptor. Proc Natl Acad Sci USA 2010;107: 16759-16765.

$12 \mathrm{Hu}$ R, Isaacs WB, Luo J. A snapshot of the expression signature of androgen receptor splicing variants and their distinctive transcriptional activities. Prostate 2011;71:1656-1667.

13 Zhang X, Morrissey C, Sun S, et al. Androgen receptor variants occur frequently in castration resistant prostate cancer metastases. PLoS ONE 2011;6:e27970.

$14 \mathrm{Qu}$ Y, Dai B, Ye D, et al. Constitutively active AR-V7 plays an essential role in the development and progression of castration-resistant prostate cancer. Sci Rep 2015;5:7654.

15 Antonarakis ES, Lu C, Wang $\mathrm{H}$, et al. AR-V7 and resistance to enzalutamide and abiraterone in prostate cancer. N Engl J Med 2014;371:1028-1038.

16 Welti J, Rodrigues DN, Sharp A, et al. Analytical validation and clinical qualification of a new immunohistochemical assay for androgen receptor splice variant-7 protein expression in metastatic castrationresistant prostate cancer. Eur Urol 2016;70:609-610.

17 Antonarakis ES, Lu C, Luber B, et al. Androgen receptor splice variant 7 and efficacy of taxane chemotherapy in patients with metastatic castrationresistant prostate cancer. JAMA Oncol 2015;1:582-591.

18 Onstenk W, Sieuwerts AM, Kraan J, et al. Efficacy of cabazitaxel in castration-resistant prostate cancer is independent of the presence of AR-V7 in circulating tumor cells. Eur Urol 2015;68:939-945.

19 Scher HI, Morris MJ, Stadler WM, et al. Trial design and objectives for castration-resistant prostate cancer: updated recommendations from the prostate cancer 
clinical trials working group 3. J Clin Oncol 2016;34: 1402-1418.

20 Geethakumari PR, Cookson MS, Kelly WK. The evolving biology of castration-resistant prostate cancer: review of recommendations from the prostate cancer clinical trials working group 3. Oncology 2016;30: 187-195.

21 Therasse P, Arbuck SG, Eisenhauer EA, et al. New guidelines to evaluate the response to treatment in solid tumors. European Organization for Research and Treatment of Cancer, National Cancer Institute of the United States, National Cancer Institute of Canada. J Natl Cancer Inst 2000;92:205-216.
22 Hussain M, Tangen CM, Higano C, et al. Absolute prostatespecific antigen value after androgen deprivation is a strong independent predictor of survival in new metastatic prostate cancer: data from Southwest Oncology Group Trial 9346 (INT-0162). J Clin Oncol 2006;24:3984-3990.

23 Nuzzo PV, Rubagotti A, Zinoli L, et al. Prognostic value of stromal and epithelial periostin expression in human prostate cancer: correlation with clinical pathological features and the risk of biochemical relapse or death. BMC Cancer 2012;12:625.

24 Choudhury AD, Eeles R, Freedland SJ, et al. The role of genetic markers in the management of prostate cancer. Eur Urol 2012;62:577-587.

Supplementary Information accompanies the paper on Modern Pathology website (http://www.nature.com/ modpathol) 